\title{
Antidepressiva und Suizidalität
}

\author{
U. Hegerl \\ Kompetenznetz Depression, Suizidalität, München, Deutschland
}

\section{Hintergrund}

Die Wirksamkeit der Antidepressiva bei der Behandlung depressiver Patienten ist durch zahlreiche und methodisch recht sorgfältige Studien gut belegt. Zu Verunsicherung der Patienten und auch mancher Ärzte hat jedoch geführt, dass in der Öffentlichkeit in den letzten Wochen verstärkt diskutiert wurde, ob Antidepressiva Suizide und Suizidversuche auslösen können.

Ausgelöst wurde die gegenwärtige Diskussion durch kritische Stellungnahmen von britischen und amerikanischen Arzneimittelbehörden zur Antidepressiva-Behandlung von Depressionen bei Kindern und Jugendlichen. Diese kritischen Äußerungen basieren vor allem darauf, dass die Wirksamkeit von Antidepressiva bei Kindern und Jugendlichen wenig untersucht ist und in mehreren Studien die Wirksamkeit gegenüber einem Scheinmedikament (Placebo) nicht belegt werden konnte. Hinzu kam, dass unter der Behandlung mit SSRI (Selektive Serotonin-Wiederaufnahmehemmer) bei Kindern und Jugendlichen das Auftreten suizidaler Impulse beobachtet wurde. Auch wenn es im Rahmen dieser Studien nicht zu Suiziden kam, so wurde diese Beobachtung vor dem Hintergrund der ungenügenden Wirksamkeitsbelege zum Anlass genommen, vor dem breiteren Einsatz von Antidepressiva bei Kindern und Jugendlichen zu warnen.

In der sich entwickelnden Diskussion wurde das Thema dann auch auf die Gruppe der Erwachsenen ausgeweitet und kontrovers diskutiert. In dieser Diskussion wurde in den Medien, aber auch von professioneller Seite vor allem die mögliche Suizid induzierende Wirkung von SSRI in den Vordergrund gerückt, z.B. unter Bezug auf einzelne Fallberichte aus den 1990er Jahren, in denen unter einer Behandlung mit SSRI eine verstärkte Suizidalität beobachtet worden war. Zum Teil wurde gar vor einer eigenständigen Suizid induzierenden Wirkung der SSRI gewarnt. Damit wurden zahlreiche Patienten und Ärzte, die mit der Datenlage nicht vertraut sind, verunsichert. Hinzu kam der Suizid einer Frau wenige Tage nach Absetzen eines hoch dosierten Antidepressivums. Die Frau hatte als gesunde Probandin an einer Medikamentenstudie teilgenommen.

\section{Überlegungen und Fakten}

Für die Einschätzung und richtige Gewichtung derartiger Berichte müssen die folgenden Überlegungen und Fakten berücksichtigt werden:

- Eine neue, bisher nur auf Kongressen vorgestellte Studie aus Schweden [Isacsson et al.] erlaubt die Abschätzung der Gefahr von SSRI hinsichtlich Suizidalität. Über viele Jahre hinweg wurden in Schweden systematisch bei allen Menschen, die sich das Leben genommen hatten, Medikamentenanalysen durchgeführt. In der Altersgruppe bis 14 Jahre kam es zu 52 Suiziden. Bei 5 dieser Suizidopfer waren Antidepressiva nachweisbar, bei keinem einzigen jedoch ein SSRI, und dies obwohl in dieser Altersgruppe SSRI am häufigsten zum Einsatz kommen (80\%)! Die manchmal reißerisch verstärkte Sorge, dass durch SSRI Kinder in den Suizid getrieben werden, kann demnach zumindest für Schweden sehr klar ausgeschlossen werden.

- In zahlreichen Studien an Erwachsenen konnte gezeigt werden, dass es unter Behandlung mit SSRI oder anderen Antidepressiva zum Abklingen von Suizidgedanken und -impulsen kommt.

- Depressionen gehen mit großem Leidensdruck und Hoffnungslosigkeit einher, so dass sich bei einem Großteil der Betroffenen suizidale Gedanken und Impulse einstellen, die nicht selten auch tatsächlich in suizidale Handlungen einmünden. Manche Betroffene mit schwersten Depressionen werden nur durch ihre Antriebslosigkeit an der Aus-

\begin{tabular}{llll}
\hline KARGER & @ 2004 S. Karger GmbH, Freiburg & & Prof. Dr. Ulrich Hegerl \\
Fax +497614520714 & Accessible online at: & Kompetenznetz Depression, Suizidalität \\
$\begin{array}{ll}\text { E-mail Information@Karger.de } \\
\text { www.karger.com }\end{array}$ & www.karger.com/ver & Psychiatrische Klinik der LMU München \\
& & Nußbaumstraße 7, D-80336 München \\
& & Tel. 089/5160-5553, Fax -5557 \\
& E-mail Anke.Schlee@med.uni-muenchen.de; www.kompetenznetz-depression.de
\end{tabular}


führung ihrer suizidalen Impulse gehindert. Bei diesen hoch gefährdeten Patienten kann auch die Einleitung einer Behandlung mit Risiken verbunden sein, da die Patienten mit Eintritt der ersten Besserung wieder genügend Antrieb erhalten, um ihre suizidalen Impulse umzusetzen. Dieses Risiko ist bekannt und gilt generell für die Phase der Besserung, gleich ob unter Antidepressiva, Psychotherapie oder in Folge des Spontanverlaufs. Dass dieses Risiko, wie bisweilen postuliert, unter der Antidepressivagruppe der SSRI größer als unter eher dämpfenden Antidepressiva ist, ist theoretisch nicht unplausibel. SSRI können den Antrieb steigern und bei einzelnen Patienten eine innere Unruhe hervorrufen. Die Daten sprechen jedoch dagegen, wie im nächsten Punkt gezeigt.

- Von Khan et al. [2003] wurden rückwirkend die Studiendaten der amerikanischen Zulassungsbehörde für Medikamente (FDA) ausgewertet. In diesen Studien wurden mehr als 58000 (!) Patienten mit Antidepressiva behandelt und alle ernsthaften Vorkommnisse genau dokumentiert. Patienten mit ernsthafter Suizidalität wurden in diese Studien aus ethischen Gründen nicht eingeschlossen. Das Suizidrisiko unter SSRI unterschied sich nun nicht von dem anderer Antidepressiva inklusive der älteren tri- und tetrazyklischen Antidepressiva (TZA). Hiermit kann so gut wie ausgeschlossen werden, dass SSRI in nennenswerter Weise Suizidalität neu hervorrufen. Wäre dies der Fall, so müsste sich dies in den Suizidraten zeigen. Allerdings ist auch festzuhalten, dass sich in diesen Studien die Suizidraten unter Placebo ebenfalls nicht von denen unter einem Antidepressivum unterschieden.

- In verschiedenen Ländern ist ein Zusammenhang zwischen der deutlichen Zunahme der Antidepressiva-Verschreibungen und Abnahme der Suizidraten gefunden worden. Derartige Zusammenhänge sind kein Beweis für eine suizidpräventive Wirkung der Antidepressiva, sie sind jedoch schwer mit der Vorstellung zu vereinbaren, dass Antidepressiva das Suizidrisiko erhöhen.

- Depressiv Erkrankte neigen dazu, Veränderungen in ihrem Befinden und ihrer Umwelt äußerst negativ und als Ausdruck der Hoffnungslosigkeit der Situation zu interpretie- ren. Bei schwer Erkrankten genügen oft auch kleinste Anlässe als Anstoß zur Ausführung suizidaler Handlungen. Ein derartiger Anlass können natürlich auch alle unter Antidepressiva auftretenden Nebenwirkungen sein, seien dies Mundtrockenheit, Übelkeit, innere Unruhe oder anderes. Vor diesem Hintergrund ist es nicht verwunderlich, dass es auch Berichte über einzelne Patienten mit Hinweisen auf einen Zusammenhang zwischen Beginn einer Anti-depressivabehandlung und verstärkter Suizidalität gibt. Derartige Berichte könnten auch zu vielen anderen Ereignissen wie zum Beispiel dem Beginn einer Psychotherapie geliefert werden.

\section{Fazit}

Bei schweren Depressionen ist Suizidalität ein ständiger dunkler Begleiter. Zu Beginn der Behandlung einer schweren depressiven Episode ist besondere Aufmerksamkeit nötig, da die antidepressive Wirkung der Pharmako- oder Psychotherapie oft erst nach 2-4 Wochen deutlich wird, da auch leichtere Nebenwirkungen von Patienten im Rahmen der depressiven Erkrankung als dramatische Verschlechterung fehlinterpretiert werden können und da mit Besserung des Antriebs suizidale Impulse leichter umgesetzt werden können. Dies gilt für alle Antidepressiva, aber auch für Psychotherapie. Spekulationen, dass SSRI in besonderem Maße die Gefahr der Suizidinduktion bergen, sind nicht nur nicht belegt, sondern können durch neuere Untersuchungen weitgehend ausgeschlossen werden. Antidepressiva können depressive Episoden zum Abklingen bringen und insbesondere das Risiko des Wiederauftretens reduzieren. Dies ist bei Erwachsenen unbestritten. Da suizidale Handlungen im Rahmen dieser depressiven Episoden auftreten, ist es mehr als plausibel, dass durch eine konsequente Pharmakotherapie Suizide und Suizidversuche verhindert werden können, auch wenn es aus methodischen Gründen schwierig ist, dies statistisch zu belegen. Es wäre tragisch, wenn durch eine unausgewogene Diskussion in diesem Bereich Patienten verunsichert und eine oft lebensrettende Behandlung mit Antidepressiva unterbleiben würde. 